\title{
Erratum: Perturbative renormalization of neutron-antineutron operators [Phys. Rev. D 93, 016005 (2016)]
}

Michael I. Buchoff and Michael Wagman

Q (Received 28 September 2018; published 11 October 2018)

DOI: 10.1103/PhysRevD.98.079901

The first sentences of Tables I-II and the last sentence of the paragraph below Eq. (22) state that there is a $(-4)$

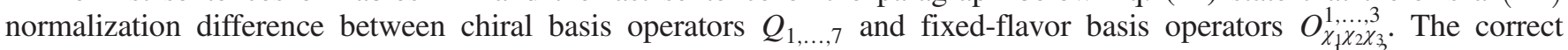
statement is that the $Q_{I}$ in the first columns of Tables I-II are proportional to the linear combinations of $O_{\chi_{1} x_{2} \chi_{3}}$ shown in the second column with proportionality factor 1 for $Q_{5}$ and (-4) for all other $Q_{I}$. Anomalous dimensions and RI-MOM to $\overline{\mathrm{MS}}$ renormalization scheme matching factors are independent of operator normalization and our results summarized in Table I are still correct as given for all operators in both bases. 